\title{
Global DNA Methylation Detection System Using MBD-Fused Luciferase Based on Bioluminescence Resonance Energy Transfer Assay
}

\author{
Wataru Yoshida,* Yuji Baba, and Isao Karube \\ School of Bioscience and Biotechnology, Tokyo University of Technology, 1404-1 Katakuramachi, Hachioji, Tokyo 192-0982, \\ Japan
}

ABSTRACT: DNA methylation plays an important role in the regulation of gene expression. In normal cells, transposable elements that constitute approximately $45 \%$ of the human genome are highly methylated to silence their expression. In cancer cells, transposable elements are hypomethylated; therefore, global DNA methylation level is considered as a biomarker for cancer diagnostics. In this study, a homogeneous assay for measuring global DNA methylation level based on bioluminescence resonance energy transfer (BRET) was developed using methyl-CpG binding domain (MBD)-fused luciferase. In this assay, the MBD-luciferase recognizes methylated CpG, thus, BRET between the luciferase and fluorescent DNA intercalating dye is detected. We demonstrated that the BRET signal depended on the DNA methylation level of the target DNA. Moreover, the BRET signal was correlated with the LINE1 DNA methylation level on human genomic DNA, as determined by the bisulfite method. These results indicate that the global DNA methylation level of human genomic DNA could be detected simply by measuring the BRET signal. 
Table S1. DNA sequences used in this study.

\begin{tabular}{ll}
\hline Name & Sequence $\left(5^{\prime}-3^{\prime}\right)$ \\
\hline Forward primer for MBD & TCTCATATGTGGAGCCATCCGCAGTTTGAAAAGGCTGAGGACTGGCTGGACT \\
Reverse primer for MBD & GTTGAATTCGCTGGCAACCGCCACGG \\
Methylated DNA_Top & Biotin-AAAAAACAGGATXGACGACGTACCCT (X= methylated cytosine) \\
Methylated DNA_Bottom & AGGGTACGTCGTXGATCCTG (X= methylated cytosine) \\
Unmethylated DNA_Top & Biotin-AAAAAACAGGATCGACGACGTACCCT \\
Unmethylated DNA_Bottom & AGGGTACGTCGTCGATCCTG \\
LINE1 bisulfite forward & GYGTAAGGGGTTAGGGAGTTTTT (Y= T or C) \\
primer & \\
LINE1 bisulfite reverse & AACRTAAAACCCTCCRAACCAAATATAAA ( R= A or G) \\
primer & \\
\hline
\end{tabular}


ATGTGGAGCCATCCGCAGTTTGAAAAGGCTGAGGACTGGCTGGACTGCCCGGCCCTGGGCCCTGGCTGGAAG CGCCGCGAAGTCTTTCGCAAGTCAGGGGCCACCTGTGGACGCTCAGACACCTATTACCAGAGCCCCACAGGA GACAGGATCCGAAGCAAAGTTGAGCTGACTCGATACCTGGGCCCTGCGTGTGATCTCACCCTCTTCGACTTCA AACAAGGCATCTTGTGCTATCCAGCCCCCAAGGCCCATCCCGTGGCGGTTGCCAGCGAATTCAGACCAATGGTT TTCATGGAGAACGATGAAAATATTGTGTATGGTCCTGAACCATTTTACCCTATTGAAGAGGGATCTGCTGGAGC ACAATTGCGCAAGTATATGGATCGACATGCAAAACTTGGAGCAATTGCTTTTACTAACGCACTTACCGGTGTCG ATTATACGTACGCCGAATACTTAGAAAAATCATGCTGTCTAGGAGAGGCTTTAAAGAATTATGGTTTGGTTGTTC ATGGAAGAATTGCGTTATGCAGTGAAAACTGTGAAGAGTTCTTTATTCCTGTATTAGCCGGTTTATTTATAGGTG TCGGTGTGGCTCCAACTAATGAGATTTACACTCTACGTGAATTGGTTCACAGTTTAGGCATCTCTAAGCCAACAA TTGTATTTAGTTCTAAAAAAGGATTAGATAAAGTTATAACTGTACAAAAAACGGTAACTGCTATTAAAACCATTG TTATATTGGACAGCAAAGTGGATTATAGAGGTTATCAATCCATGGACAACTTTATTAAAAAAAACACTCCACAAG GTTTCAAAGGATCAAGTTTTAAAACTGTAAAAGTTAACCGCAAAGAACAAGTTGCTCTTATAATGAACTCTTCG GGTTCAACCGGTTTGCCAAAAGGTGTGCAACTTACTCATGAAAATTTGGTCACGCGTTTTTCTCACGCTAGAGA TCCAATTTATGGAAACCAAGTTTCACCAGGCACGGCTATTTTAACTGTAGTACCATTCCATCATGGTTTTGGTAT GTTTACTACTTTAGGCTATCTAACTTGTGGTTTTCGTATTGTCATGTTAACGAAATTTGACGAAGAGACTTTTTTA AAAACACTGCAAGATTACAAATGTTCAAGCGTTATTCTTGTACCGACTTTGTTTGCAATTCTTAATAGAAGTGAA TTACTCGATAAATATGATTTATCAAATTTAGTTGAAATTGCATCTGGCGGAGCACCTTTATCTAAAGAAATTGGTG AAGCTGTTGCTAGACGTTTTAATTTACCGGGTGTTCGTCAAGGCTATGGTTTAACAGAAACAACCTCTGCAATTA TTATCACACCGGAAGGCGATGATAAACCAGGTGCTTCTGGCAAAGTTGTGCCATTATTTAAAGCAAAAGTTATC GATCTTGATACTAAAAAAACTTTGGGCCCGAACAGACGTGGAGAAGTTTGTGTAAAGGGTCCTATGCTTATGAA AGGTTATGTAGATAATCCAGAAGCAACAAGAGAAATCATAGATGAAGAAGGTTGGTTGCACACAGGAGATATT GGGTATTACGATGAAGAAAAACATTTCTTTATCGTGGATCGTTTGAAGTCTTTAATCAAATACAAAGGATATCAA GTACCACCTGCTGAATTAGAATCTGTTCTTTTGCAACATCCAAATATTTTTGATGCCGGCGTTGCTGGCGTTCCA GATCCTATAGCTGGTGAGCTTCCGGGAGCTGTTGTTGTACTTAAGAAAGGAAAATCTATGACTGAAAAAGAAGT AATGGATTACGTTGCTAGTCAAGTTTCAAATGCAAAACGTTTGCGTGGTGGTGTCCGTTTTGTGGACGAAGTAC CTAAAGGTCTCACTGGTAAAATTGACGGTAAAGCAATTAGAGAAATACTGAAGAAACCAGTTGCTAAGTGA

Figure S1. Sequence of Streptag-MBD-luciferase. The Streptag, MBD, and luciferase sequences are highlighted in green, blue, and red, respectively. 


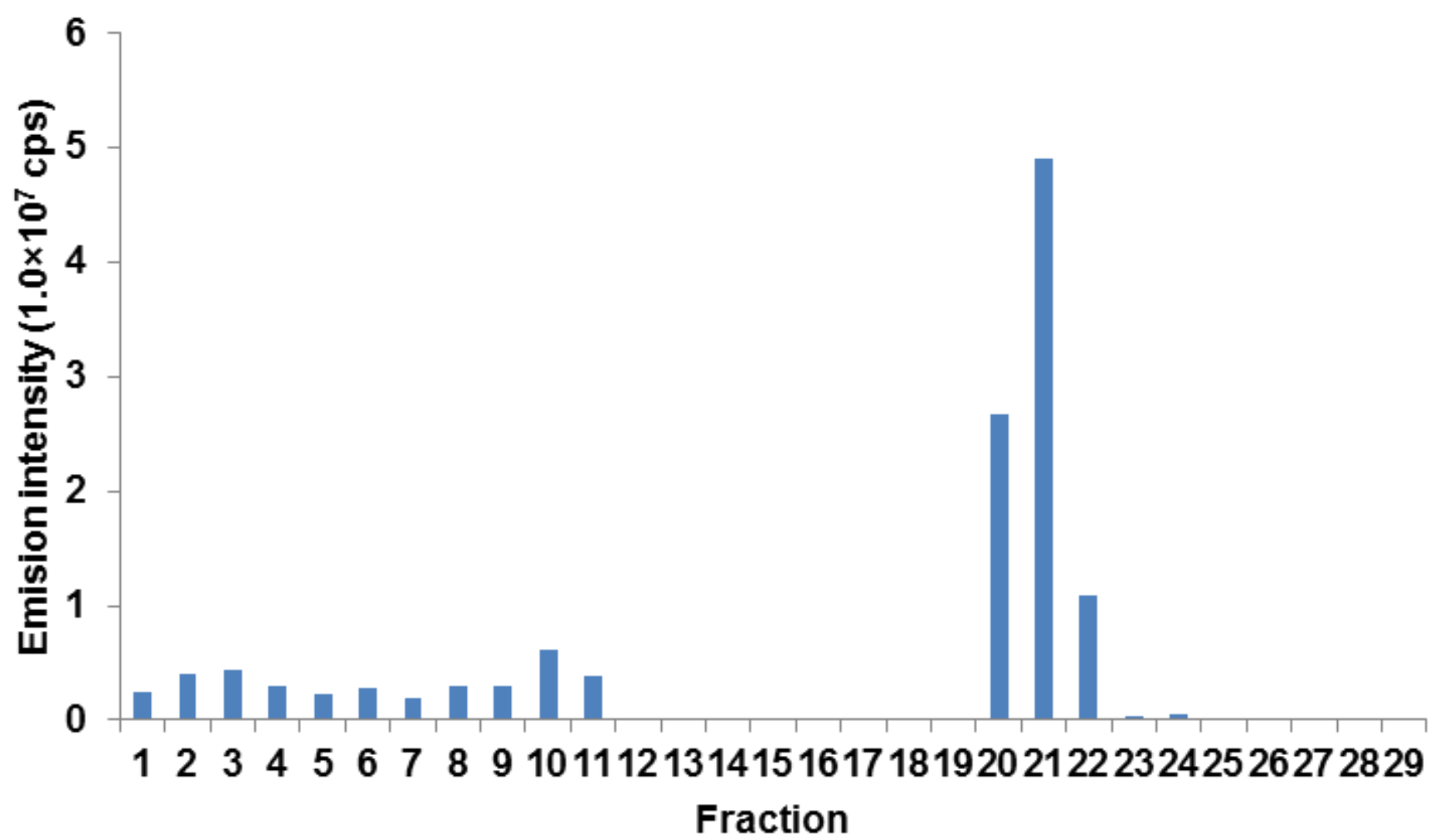

Figure S2. Luciferase activity of each fraction in the purification of MBD-luciferase. The luciferase activities of the flow-through fractions (1-9), wash fractions (10-19), and elution fractions (20-29) were measured using $10 \mu \mathrm{L}$ of the samples and $100 \mu \mathrm{L}$ of PicaGene. In all steps, $1.0 \mathrm{~mL}$ of each fraction was collected. 


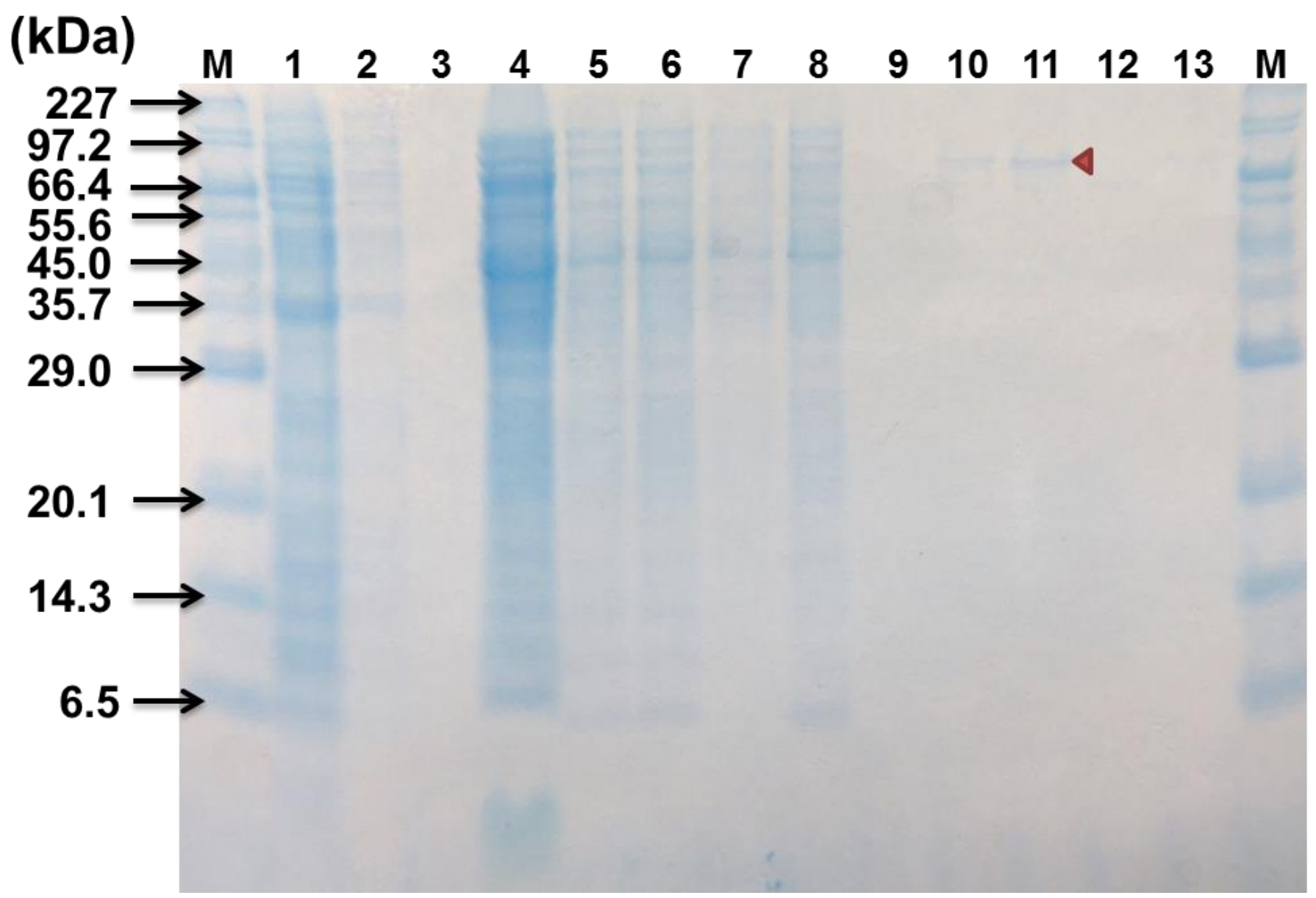

Figure S3. SDS-PAGE of the purified MBD-luciferase (71 kDa). M: protein marker; 1: insoluble fraction; 2: 10-fold-diluted insoluble fraction; 3: 100-fold-diluted insoluble fraction; 4: water-soluble fraction; 5: 10-fold-diluted water-soluble fraction; 6: filtered water-soluble fraction; 7: flow-through fraction (\#9 fraction in Figure S1); 8: wash fraction (\#10 fraction in Figure S1); 9: wash fraction (\#19 fraction in Figure S1); 10: elution fraction (\#20 fraction in Figure S1); 11: elution fraction (\#21 fraction in Figure S1); 12: elution fraction (\#22 fraction in Figure S1); 13: elution fraction (\#23 fraction in Figure S1). 


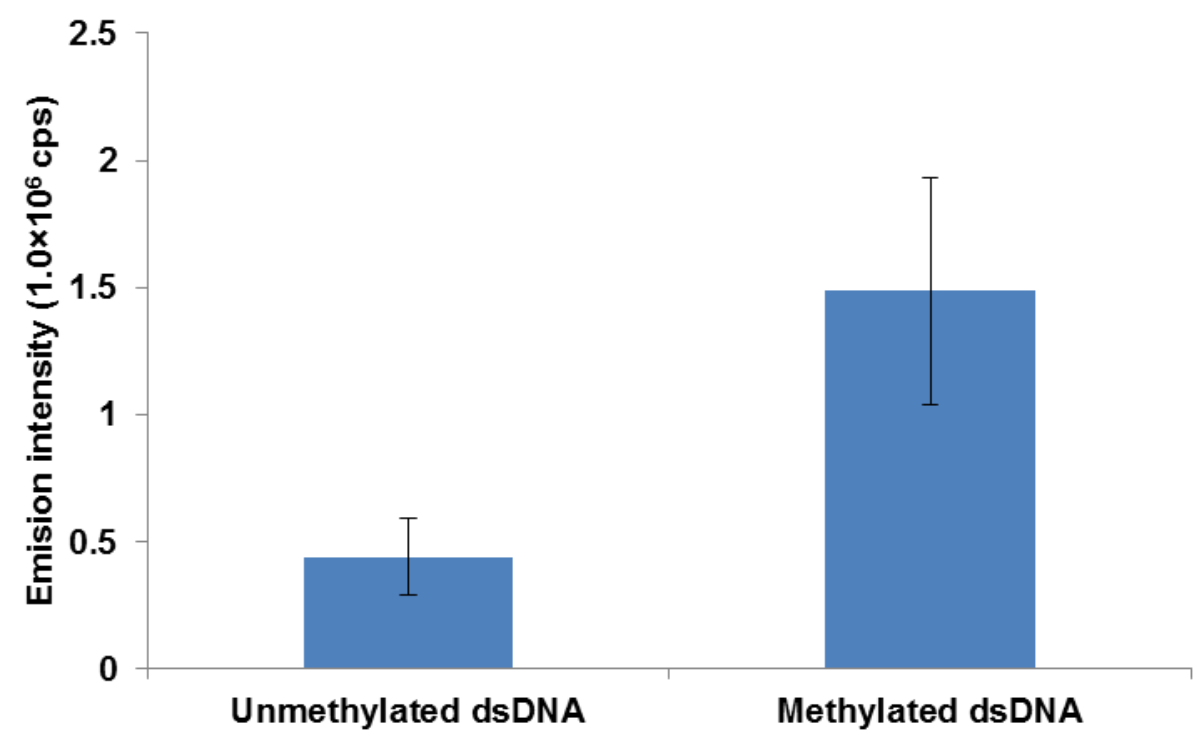

Figure S4. Analysis of the binding of MBD-luciferase to methylated dsDNA. The DNA ( $250 \mathrm{pmol})$ was added to the streptavidin-immobilized plate. After washing the well with $200 \mu \mathrm{L}$ of washing buffer (PBS, 0.05\% Tween 20), $200 \mu \mathrm{L}$ of MBD-luciferase (34 nM) was added and then incubated for 30 min at RT. After washing the well, luciferase activity was measured by the addition of $100 \mu \mathrm{L}$ of PicaGene (mean $\pm \mathrm{SD}, \mathrm{N}=3$ ). 


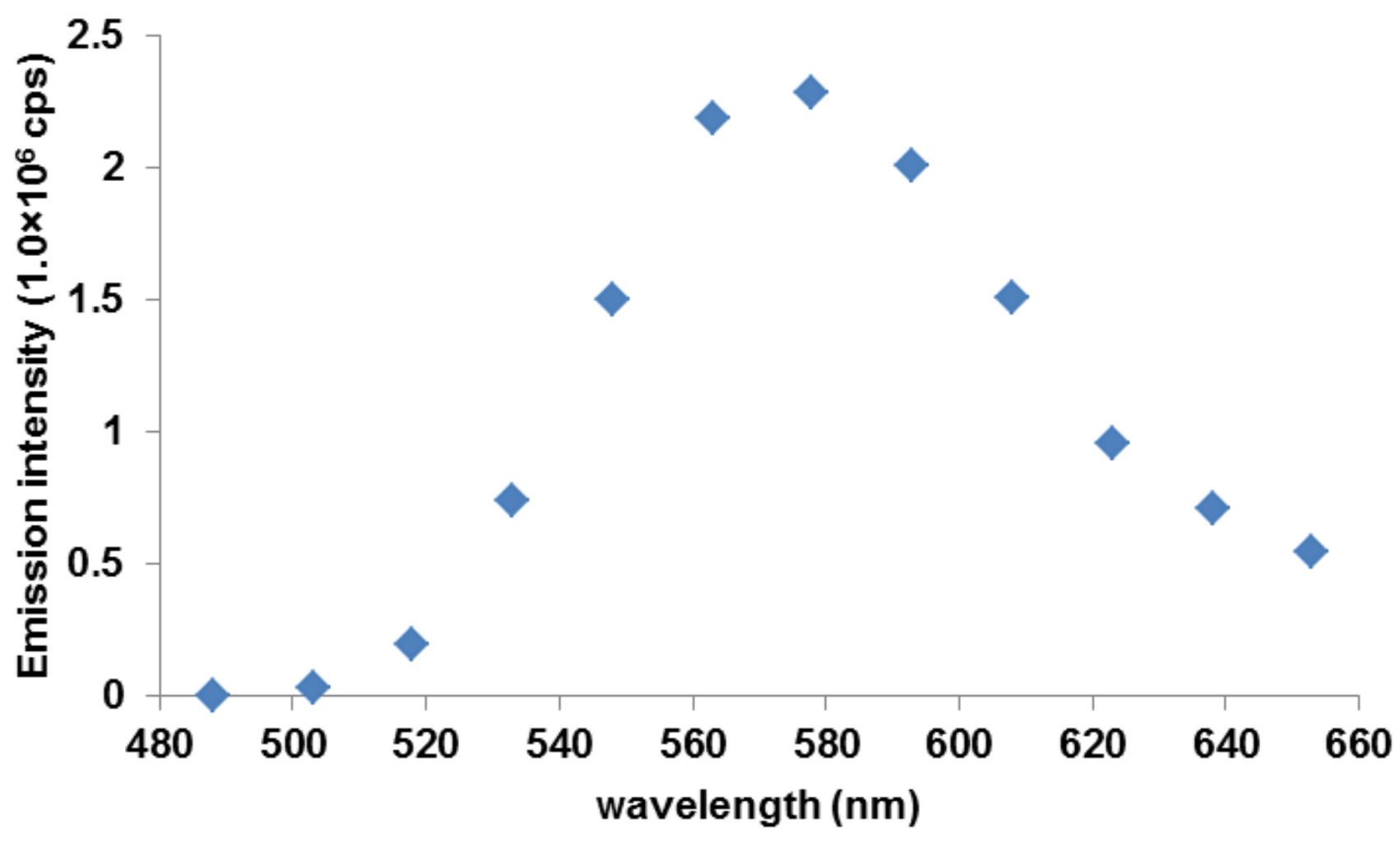

Figure S5. The spectrum of MBD-luciferase bioluminescence. The emission intensity of $17 \mathrm{nM}$ MBD-luciferase was measured in PBS buffer using $50 \mu \mathrm{L}$ of PicaGene in a $100 \mu \mathrm{L}$ reaction volume. 


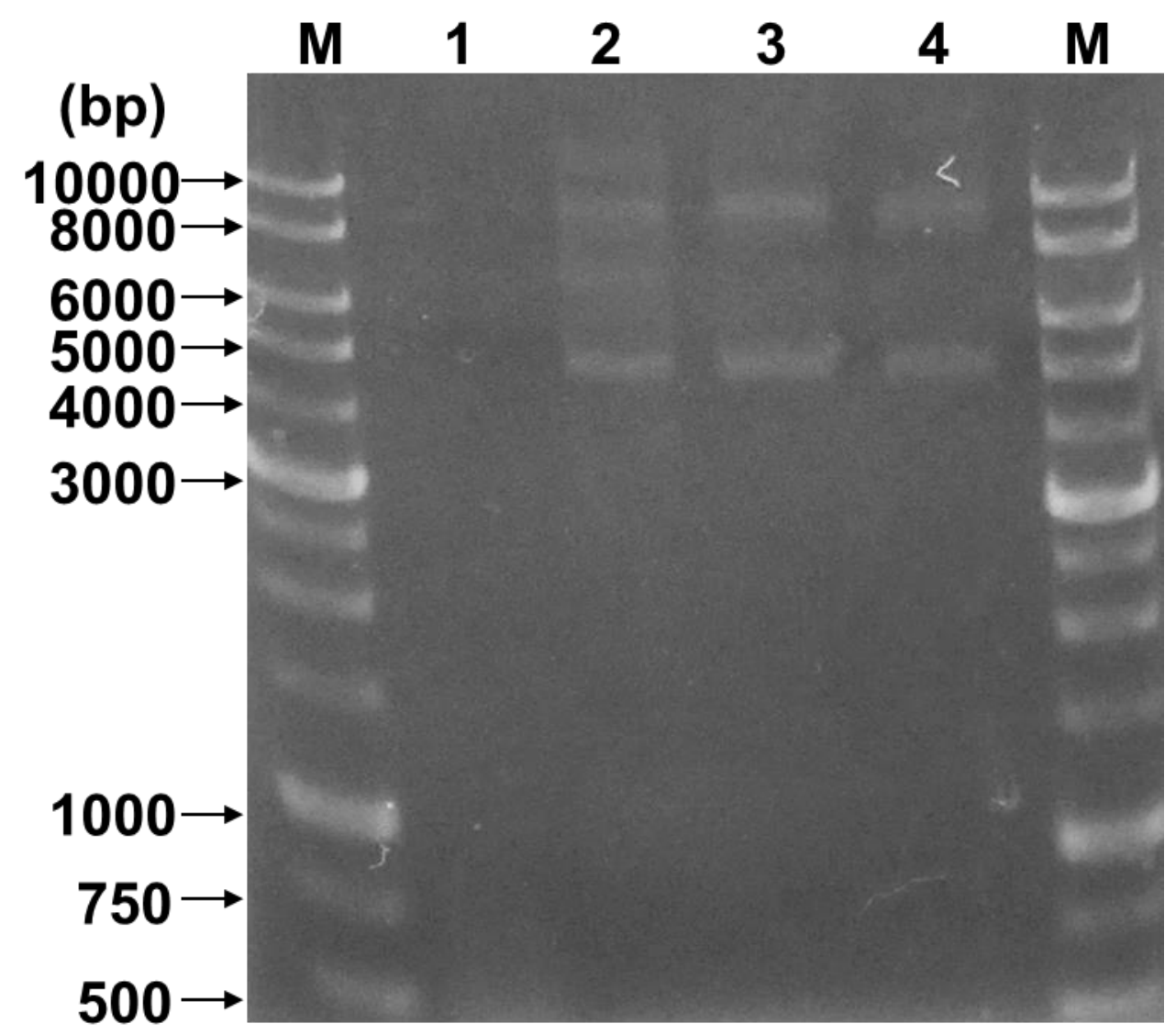

Figure S6. DNA methylation analysis of the plasmid DNA. Lane 1: unmethylated plasmid DNA treated with HpaII; lane 2: methylated plasmid DNA treated with HpaII; lane 3: unmethylated plasmid DNA; lane 4: methylated plasmid DNA. 


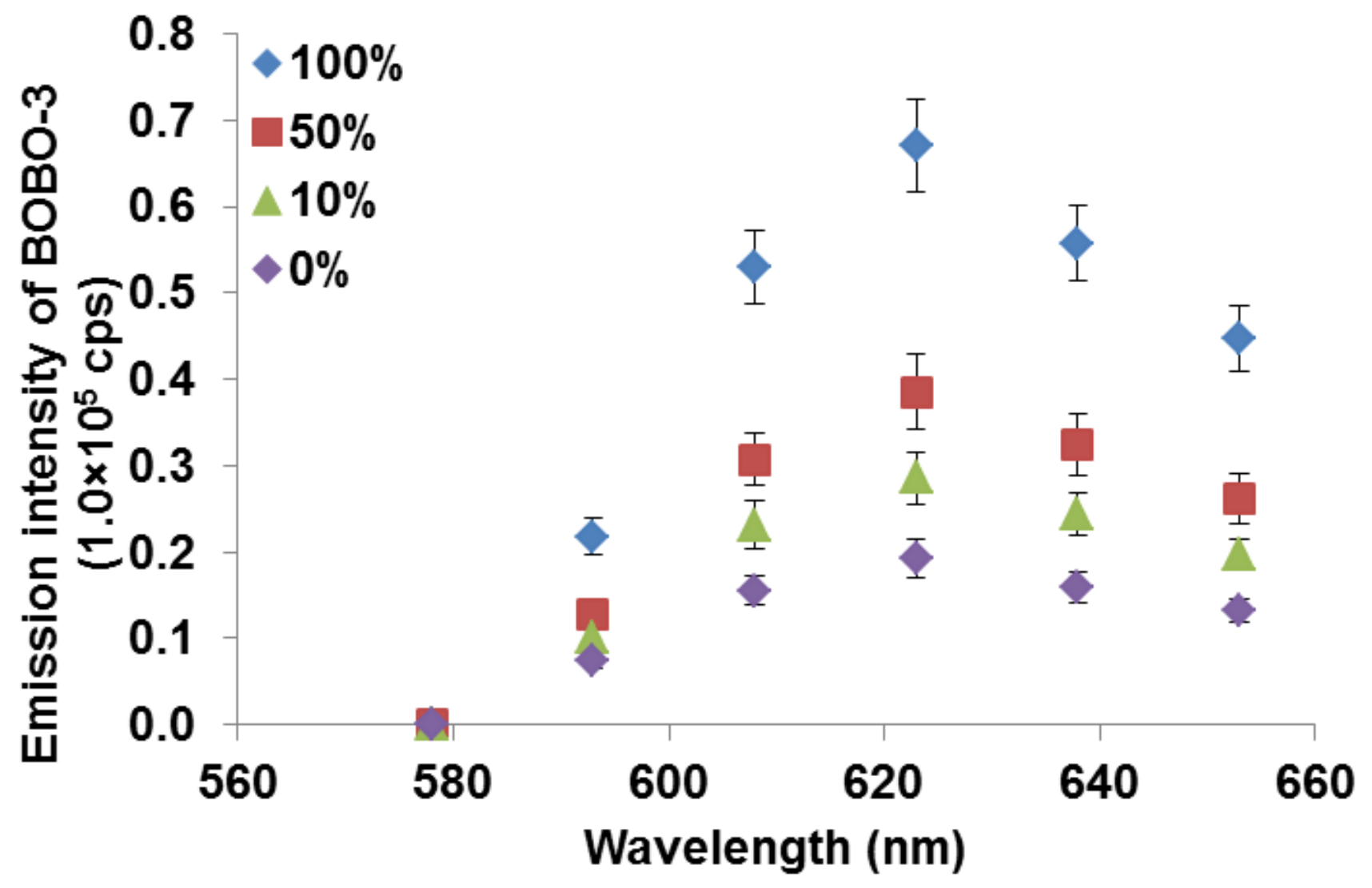

Figure S7. Emission intensity of DNA intercalating dye excited by MBD-luciferase in the presence of methylated plasmid DNAs. In the assay, $17 \mathrm{nM}$ MBD-luciferase, $1.0 \mu \mathrm{M}$ BOBO-3, and 0\%, 10\%, 50\%, and 100\% methylation of $10 \mathrm{ng} / \mu \mathrm{L}$ plasmid DNA were used. The emission intensity of BOBO-3 was calculated by subtracting the emission intensity of MDB-luciferase from the total emission intensity at each wavelength (mean $\pm \mathrm{SD}, \mathrm{N}=3$ ). 


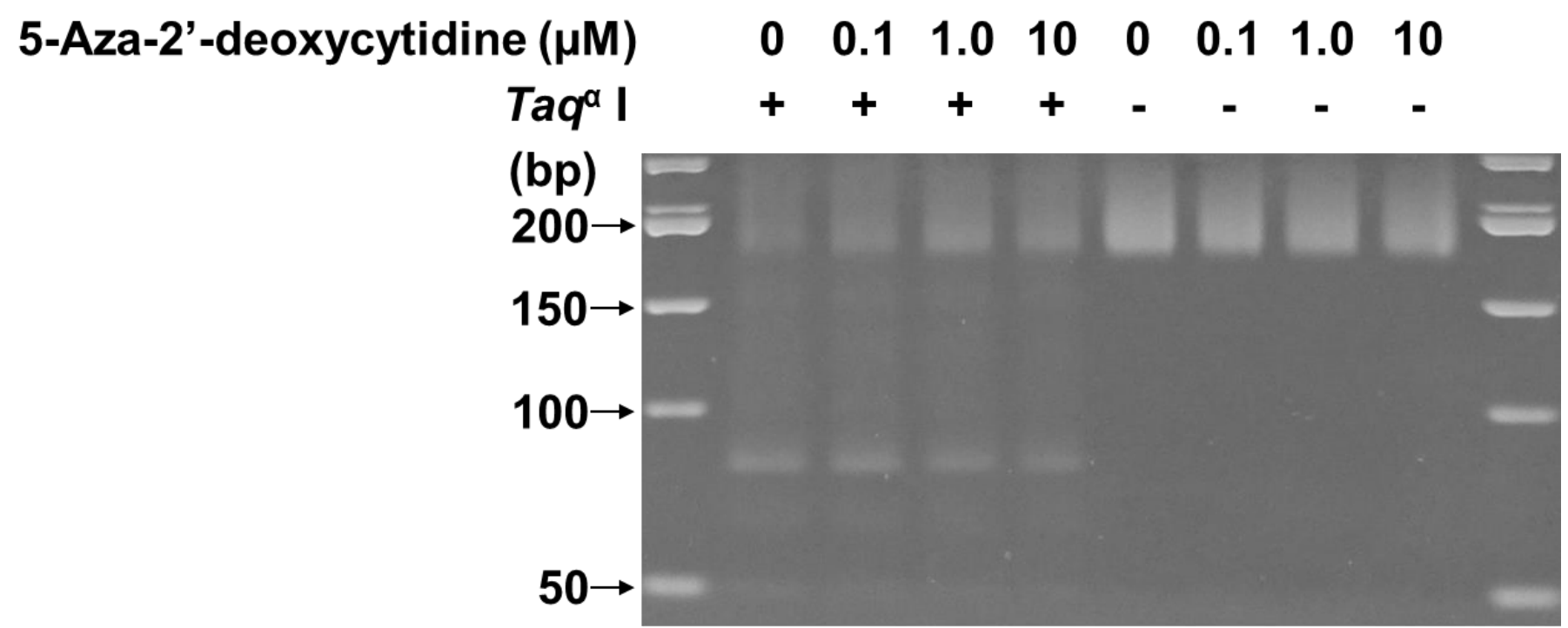

Figure S8. DNA methylation analysis of LINE1 by COBRA. DNA methylation level of LINE1 region on genomic DNA purified from HeLa cells treated with 10, 1.0, or $0.1 \mu \mathrm{M}$ 5-Aza-2'-deoxycytidine was analyzed. 


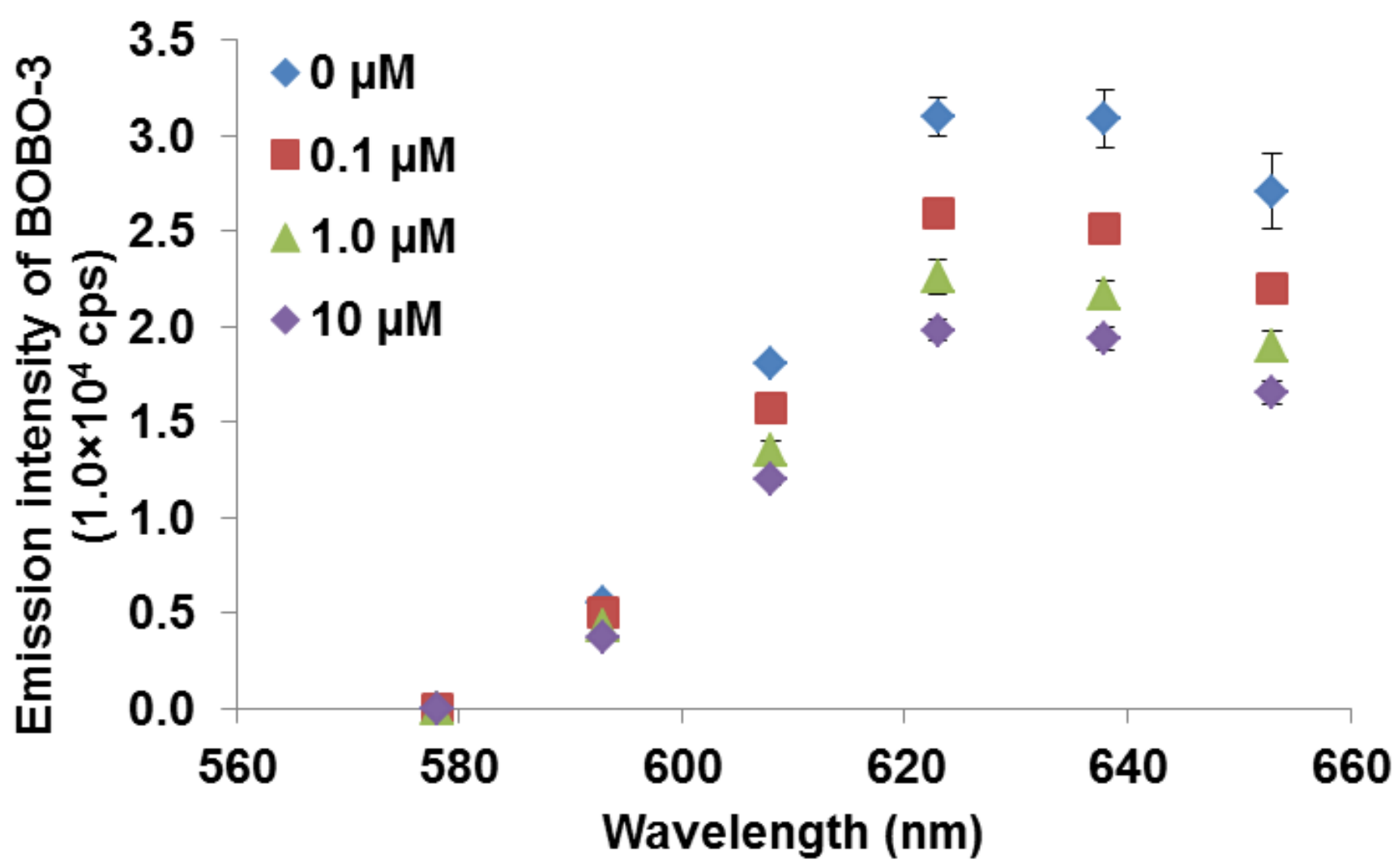

Figure S9. Emission intensity of DNA intercalating dye excited by MBD-luciferase in the presence of genomic DNA purified from HeLa cells treated with 5-Aza-2'-deoxycytidine. In the assay, $17 \mathrm{nM}$ MBD-luciferase, 1.0 $\mu \mathrm{M}$ BOBO-3, and $70 \mathrm{ng} / \mu \mathrm{L}$ genomic DNA purified from HeLa treated with 10, 1.0, or $0.1 \mu \mathrm{M} 5$-Aza-2'-deoxycytidine were used. The emission intensity of BOBO-3 was calculated by subtracting the emission intensity of MDB-luciferase from the total emission intensity at each wavelength (mean $\pm \mathrm{SD}, \mathrm{N}=3$ ). 